\title{
How can a 22-pole ion trap exhibit 10 local minima in the effective potential?
}

\author{
R. Otto ${ }^{1}$, P. Hlavenka ${ }^{1}$, S. Trippel ${ }^{1}$, J. Mikosch 1 团, K. \\ Singer $^{2}$, M. Weidemüller 1 \&, R. Wester ${ }^{1}$ \\ ${ }^{1}$ Physikalisches Institut, Universität Freiburg, Hermann-Herder-Straße 3, 79104 \\ Freiburg, Germany \\ 2 Institut für Quanteninformationsverarbeitung, Universität Ulm, \\ Albert-Einstein-Allee 11, 89081 Ulm, Germany \\ E-mail: roland.wester@physik.uni-freiburg.de
}

\begin{abstract}
The column density distribution of trapped $\mathrm{OH}^{-}$ions in a 22-pole ion trap is measured for different trap parameters. The density is obtained from position-dependent photodetachment rate measurements. Overall, agreement is found with the effective potential of an ideal 22-pole. However, in addition we observe 10 distinct minima in the trapping potential, which indicate a breaking of the 22 -fold symmetry. Numerical simulations show that a displacement of a subset of the radiofrequency electrodes can serve as an explanation for this symmetry breaking.
\end{abstract}

\footnotetext{
$\ddagger$ present address: National Research Council Canada, 100 Sussex Drive, Ottawa,ON K1A 0R6, Canada

$\S$ present address: Physikalisches Institut, Universität Heidelberg, Philosophenweg 12, 69120 Heidelberg, Germany
} 


\section{Introduction}

Multipole radiofrequency ion traps 1], in particular the 22-pole ion trap 2, are versatile devices used in laser spectroscopy $[3,4,5,6,7,8,9,10$, and investigations of chemical reaction processes [2, 11, 12, 13, of atomic and molecular ions. High order multipole traps offer a large field free region in the trap center, and therefore provide a reduced interaction time of the ions with the oscillating electric field compared to a quadrupole trap [14]. Buffer gas cooling down to cryogenic temperatures of the translational [3, 7], rotational [3, 9, and vibrational degrees of freedom [8] of trapped molecular ions has been demonstrated. This enables applications with all the advantages of low temperature experiments, such as a reduced Doppler width in spectroscopy studies and a well defined population of internal states.

Stable confinement of a single ion in the oscillating quadrupole field of a Paul trap is precisely predicted, because the Mathieu equations of motion can be solved analytically. This is different for oscillating high order multipole fields, where the equations of motion have no analytical solution. However, the movement of the ions in a fast oscillating rf-field justifies the assumption of an effective trapping potential, based on the separation of the ion motion into a smooth drift and a rapid oscillation, called micromotion [15]. This effective potential can be expressed as

$$
V^{*}(\vec{r})=\frac{1}{4} \frac{(q \vec{E}(\vec{r}))^{2}}{m \Omega^{2}}+q \Phi_{0}
$$

where $\vec{E}(\vec{r})$ denotes the electric field at the point $\vec{r}, m$ is the mass of a test particle with charge $q$ in an electric field oscillating on frequency $\Omega$ and $\Phi_{0}$ is a non-oscillating dc potential. For an ideal cylindrical multipole of the order $n$ this effective potential can be expressed as

$$
V^{*}(r)=\frac{1}{4} \frac{n^{2}\left(q V_{0}\right)^{2}}{m \Omega^{2} r_{0}^{2}}\left(\frac{r}{r_{0}}\right)^{2 n-2}
$$

where $V_{0}$ denotes the amplitude of the oscillating rf-field. For a high order multipole field ( $n=11$ for the 22-pole trap) this creates an almost box-like trapping volume with steep walls and a large field free region in the center. This is important to suppress $\mathrm{rf}$ heating when cooling ions in neutral buffer gas [14.

The number of trapped ions that can be excited with radiation depends on the local density of ions in the interaction volume with the light field. In experiments with trapped $\mathrm{Ba}^{+}$ions in an octupole trap the density has been imaged by spatially resolving the fluorescence signal [16, 17]. In this article we report on a method based on the photodetachment of trapped anions that allows us to image the density distribution of the trapped ions. In this way we measure the experimental trapping potential of a 22-pole trap with unprecedented accuracy and study its dependence on the radiofrequency field. In the next section the photodetachment tomography is explained, followed by a presentation of measured density distributions at different temperatures and $\mathrm{rf}$ amplitudes. We find ten unexpected maxima in the density distribution and analyze their origin with the help of numerical simulations in section 4

\section{Experimental procedure}

The experimental radiofrequency ion trap setup has been described in detail in Ref. [18. Its central element is a 22-pole trap [2] with good optical access along the trap 
axis. In the present tomography experiments, we have used an ensemble of stored $\mathrm{OH}^{-}$anions cooled to a translational temperature of either 170 or $300 \mathrm{~K}$. The use of negative ions allows us to perform photodetachment measurements, were the anions are depleted by a laser beam propagating parallel to the symmetry axis of the trap [19, 20. The photodetachment process

$$
\mathrm{OH}^{-}+\mathrm{h} \nu \rightarrow \mathrm{OH}+\mathrm{e}^{-}
$$

with $h \nu>1.83 \mathrm{eV}$ yields a depletion rate proportional to the photodetachment cross section and to the overlap of the ion column density with the photon flux. By scanning the position of the laser beam and measuring the depletion rate at each position the relative ion column density distribution is obtained. For small ion densities, where the Coulomb interaction between the ions can be neglected, the depletion rate at the transverse position $(x, y)$ can be expressed as

$$
k(x, y)=\sigma_{p d} F_{L} \rho(x, y) .
$$

It depends only on the total photon flux $F_{L}$, the photodetachment cross section $\sigma_{p d}$ and the single particle column density $\rho(x, y)$, reflecting the spatial overlap of the laser beam with the ion distribution [19. As the latter is normalized to unity, we can write it as

$$
\rho(x, y)=\frac{k(x, y)}{\int_{S} k(x, y) d S}
$$

Thus, a full two-dimensional tomography scan of the trapped ions can be used to map the entire ion column density in the trap. If $k(x, y)$ is measured for the whole ion distribution, also the absolute photodetachment cross section can be obtained [20.

Ions are produced in a pulsed supersonic expansion of a suitable precursor gas, crossed by a $1 \mathrm{keV}$ electron beam. For the $\mathrm{OH}^{-}$production we use a mixture of Ar $/ \mathrm{NH}_{3} / \mathrm{H}_{2} \mathrm{O}(88 \%, 10 \%, 2 \%)$ to create $\mathrm{NH}_{2}^{-}$. A rapid chemical conversion by water forms the $\mathrm{OH}^{-}$anions in the source. A bunch of $~ 500$ mass-selected ions is loaded into the trap, which is enclosed by a copper housing that is temperature-variable between 8 and $300 \mathrm{~K}$. A typical He buffer gas density of $1 \times 10^{14} \mathrm{~cm}^{-3}$, employed for all temperatures, is enhanced by a buffer gas pulse during the ion injection. The trap is operated with $\Omega=2 \pi \times 5 \mathrm{MHz}$ radiofrequency and different rf amplitudes. Along the axial direction ions are confined by end electrodes biased to $-2 \mathrm{~V}$. To allow good thermalization of the ions, a storage period of $200 \mathrm{~ms}$ is inserted before the laser beam is switched on. After a given storage and laser interaction time the current signal, proportional to the number of ions that survived the interaction with the photodetachment laser, is detected with a microchannel plate.

The two-dimensional tomography scans are performed under the same experimental conditions as in Ref. [20]. A free-running continuous wave diode laser at $661.9 \mathrm{~nm}$ (Mitsubishi ML1J27, 100mW, spectral width $0.7 \mathrm{~nm}$ FWHM) has been employed. It is imaged into the trap and scanned in the $(x, y)$ plane, perpendicular to the symmetry axis of the trap, by moving the imaging lense on a mesh with $0.25 \mathrm{~mm}$ point spacing with a computer-controlled two-dimensional translation stage. The mesh spacing is comparable to the $1 / e^{2}$ radius of the laser in the trap of $350 \mu \mathrm{m}$. For each laser position the photodetachment depletion rate is obtained in a storage time interval of $0.2-2 \mathrm{~s}$ by fitting an exponential decay to the ion current signal, reduced by typically one percent due to the background loss rate. The data are averaged over typically 4-8 scans. Within each scan the mesh points are accessed in random order to avoid systematic drifts. 


\section{Tomography of the trapping potential}

Fig. 17a shows a tomography scan of $\mathrm{OH}^{-}$anions in the 22 -pole trap at $300 \mathrm{~K}$ with the rf amplitude set to $160 \mathrm{~V}$. The figure also contains the sketched arrangement of the trap's copper housing mounted on the coldhead of the cryostat, the position of the 22 rf electrodes and the axial end electrodes. Fig. 1b is a zoom of the scan which more clearly shows the measured ion density distribution. Every pixel of the histogram here represents a fitted photodetachment depletion rate $k(x, y)$ (see previous section) and is proportional to the single-particle probability density $\rho(x, y)$ along a column parallel to the $z$-axis. As can be seen the ion distribution as a first approximation can be considered circularly symmetric and constant in the center region of the trap, whereas it drops to zero when the ions reach the outer regions of the trapping volume. This distribution directly visualizes the overall storage properties of a 22-pole ion trap with a flat potential in the center and steep walls. Note that the ion density drops to zero already for smaller radii than the end electrode (solid line in Fig. 1b), indicating that clipping of the laser at the end electrode is not affecting the measured density distribution. For smaller rf amplitudes the ion density distribution would extend to larger radii and could not be fully probed by the photodetachment tomography. For this reason we have restricted ourselves to large enough rf amplitudes in this study.

In Fig. 1. a horizontal cut through the ion distribution is shown. While in the center the distribution is relatively uniform, the population is locally enhanced by up to $40 \%$ near the edge of the ion distribution. Such a behavior has already been observed in previous measurements 19 . To study this here in more detail, the effective potential $V(x, y)$ is extracted from the local ion density $\rho(x, y)$ assuming a Boltzmann distribution for the ions in the trap

$$
\rho(x, y)=\frac{1}{Z} \exp \left(-V(x, y) / k_{\mathrm{B}} T\right),
$$

where $T$ is the absolute temperature, $k_{\mathrm{B}}$ is Boltzmann's constant, and $Z$ is the partition function. Since only the column density is measured, the resulting potential $V(x, y)$ is an average over the $z$-direction. Fig. 1 d shows a cut through the obtained effective potential for the distribution of Fig. [1 . Overall this potential compares well with the calculated potential of an ideal 22-pole potential (solid line), obtained without any free parameters from Eq. (2). Closer inspection reveals interesting features in the potential that deviate from the ideal multipole. While the potential is relatively flat in the center, it shows a distinct minimum of about $12 \mathrm{meV}$ near the left edge of the ion distribution and a weaker minimum of about $5 \mathrm{meV}$ near its right edge. It can be excluded that this change of the distribution is caused by space charge effects, because the experiments are performed with only a few hundred ions in a trap volume of about $1 \mathrm{~cm}^{3}$.

The same features of the effective potential are observed in measurements at a lower trap temperature. Fig. $2 \mathrm{a}$ shows a tomography scan at $170 \mathrm{~K}$ and the same rf amplitude as above. The ion distribution again looks circular symmetric with a distinct cutoff when the ions reach the steep walls of the trapping potential. A horizontal cut through the effective potential, obtained in the same fashion as Fig. 1 $\mathrm{d}$, is shown in Fig. 2b. The same minima as for $300 \mathrm{~K}$ are observed in the effective potential. At this lower temperature the two minima are better resolved and appear similar in depth on the left side and slightly deeper on the right side of the potential as compared to the $300 \mathrm{~K}$ tomography. 
Further substructure becomes visible in the $170 \mathrm{~K}$ density distribution. Ten clearly separated maxima in the density distribution appear almost equally spaced in angle at a radial position of about $3 \mathrm{~mm}$. According to Eq. (6) they correspond to ten localized minima in the trapping potential at this radius with a typical depth of $10 \mathrm{meV}$. These minima have not been significant in the $300 \mathrm{~K}$ ion distribution at $160 \mathrm{~V}$ rf amplitude. They become visible, however, for larger amplitudes. Fig. 3 a shows a $300 \mathrm{~K}$ ion distribution for an $\mathrm{rf}$ amplitude of $270 \mathrm{~V}$. It reveals the same ten density maxima and respective potential minima that could only be resolved at lower temperature at $160 \mathrm{~V}$.

We have studied the dependence of the depth of the ten potential minima on the rf amplitude at $300 \mathrm{~K}$. The depth of the deepest minimum is plotted in Fig. 3b. Since the effective potential is expected to depend quadratically on the rf amplitude, a fit with only a constant and a quadratic term is applied to the data (solid line in Fig. 3b). It yields an rf-independent offset of about $11 \mathrm{meV}$, which is attributed to the static potential of the end electrodes of the ion trap. These end electrodes produce a radially repulsive potential inside the trap, as discussed in Ref. 19, 21. It compares well with simulations, as shown in the next section. The ten "pockets" in the potential, however, reveal a more complex deviation from the ideal multipole description of Eq. (2). An explanation for them will also be discussed in the next section.

\section{Modeling trapping potentials of realistic multipoles}

The effective potential of a $2 n$-pole has a $2 n$-fold rotational symmetry, when averaging over one radiofrequency period. The appearance of the ten observed potential minima is therefore a clear indication for a breaking of the ideal symmetry. To investigate this effect further, the effective potential of the employed 22-pole trap has been modeled using a numerical simulation package based on a fast multipole solver [22, 23, for the boundary element problem in combination with accurate field evaluation in free space. With this method the electric field $E(r)$ can be calculated at any location inside the trap. It is converted into the effective trapping potential using Eq. (11). We have verified that the simulation of the trapping potential of an ideal 22-pole structure reproduces the effective potential of Eq. (2) on the numerical level of accuracy.

Different assumptions have been tested as origin of the observed ten potential minima, such as the influence of the shape and position of the end electrodes and of the copper housing around the trap electrodes, without showing a measurable effect on the potential. This suggests that imperfections of the trap geometry itself may be responsible. To simulate these imperfections a breaking of the ideal symmetry is introduced by displacing one half of the 22 radiofrequency electrodes by a small angle (see inset in Fig. 4a). Such a small tilt of one set of electrodes against the others occurs to be the most likely displacement during the trap assembly. Upon tilting one set of electrodes by only a few tenths of a degree the calculated effective potential of a 22 -pole trap at $160 \mathrm{~V}$ rf amplitude and $-2 \mathrm{~V}$ on the end electrodes immediately shows ten potential minima.

In Fig. 唡 the dependence of the maximum pocket depth on the tilt angle, as obtained from a series of simulations, is plotted. These simulations have been carried out for $160 \mathrm{~V} \mathrm{rf}$ amplitude. Here, an imperfection in the parallelity of only $0.2^{\circ}$ causes a pocket depth of $5 \mathrm{meV}$. The pocket depth is calculated at each angle with and without a potential of $-2 \mathrm{~V}$ applied to the static end electrodes. The end electrode voltage produces an overall quadrupole potential that pushes the ion ensemble towards larger 
radii in the trap in addition to the tilt-induced pockets. Both data sets with and without end electrode potential are described by the same quadratic increase. For the simulations with end electrode potential a constant offset of about $9 \mathrm{meV}$ is obtained, in fair agreement with the experiment value of about $11 \mathrm{meV}$.

From the measured depths of the potential minima (Fig. 3b) a value of between 3 and $5 \mathrm{meV}$ is extracted for an rf amplitude of about $160 \mathrm{~V}$, after subtracting the influence of the static end electrode (see Fig. 3b). Such an rf-induced pocket depth is obtained in the simulation for a tilt angle of between $0.15^{\circ}$ and $0.2^{\circ}$ (see Fig. 4a). Fig. 40 shows a simulated density distribution for a tilt angle of $0.15^{\circ}$. Following Eq. (6), the simulation has been performed for $\mathrm{OH}^{-}$ions that are stored at $300 \mathrm{~K}$ in the 22-pole trap with $270 \mathrm{~V}$ rf amplitude and $-2 \mathrm{~V}$ potential on the end electrodes. This simulated density distribution agrees well with the measured distribution of Fig. 3a, which has been obtained with the same trap parameters. A larger tilt angle was found to already overestimate the ten potential minima. Note that it is preferable to compare graphs of the density distributions of simulation and experiment instead of effective potentials, because the experimental potential is obtained by a logarithm of the density distribution which suppresses the fine details in the images.

When the 22-pole trap was assembled the strong influence of small displacements of the rf electrodes on the effective potential was not known. A tilt of one set of $\mathrm{rf}$ electrodes by a tenth of a degree can therefore not be excluded for our trap. Such small tilt angles already come close to the mechanical tolerances for the assembly of a 22 -pole trap in the presently used design. This shows the need to significantly improve the precision in the $\mathrm{rf}$ electrode geometry when a potential with pocket depths in the $\mu \mathrm{eV}$ range is desired.

In searching for an explanation for the observed ten minima, we have extended the electric field simulations to multipoles of different order $n$. These calculations have shown that the number of minima observed in the effective potential of a distorted multipole ion trap is directly connected to the multipole order as $N_{\text {minima }}=n-1$. Besides the above discussed tilt of one set of electrodes, other distortions of the ion trap, such as a parallel displacement of one set of rf electrodes, also introduces $n-1$ minima. The number of minima therefore seems to be a general consequence of breaking the symmetry of a multipole ion trap. We expect the minima to be related to the points in space where the superimposed time-dependent electric field of the individual multipole electrodes cancels, because according to Eq. (11) these are the global minima of the effective potential. For a perfectly symmetric multipole trap cancellation is expected only in the trap center, but for a distorted symmetry several such points are found at larger radii.

\section{Conclusion}

In this article we report on a method to directly measure the column density distribution of ions in a 22-pole ion trap using photodetachment of stored $\mathrm{OH}^{-}$. The two-dimensional tomography scans yield the effective potential averaged over the length of the trap. The measurements quantitatively confirm the overall validity of the effective potential of a 22-pole ion trap, which scales as $r^{20}$. For large rf amplitudes, however, new features in the potential have been observed in the form of ten almost equally spaced potential minima. These minima arise from the breaking of the 22fold symmetry of the trap. They can be quantitatively explained by a slight tilt of half of the multipole rf electrodes within their mechanical tolerances. Also for other 
multipole ion traps $n-1$ minima in the effective trapping potential have been found as a consequence of a broken symmetry of the trap.

This observation, which has become possible due to the high sensitivity of our photodetachment tomography scans, has implications for other spectroscopic experiments in 22-pole ion traps. In particular at cryogenic temperatures, trapped ions will reside predominantly in the ten pockets of the potential. Correspondingly, the ion density along the symmetry axis of the trap would become very small and only a small spectroscopic signal would be detected for a laser beam pointing along the trap axis. Further studies are needed to find out if ions that reside in the ten pockets may be subject to enhanced radiofrequency heating, similar to the influence of the static end electrode potential, which can increase the translational temperature by a few Kelvin [21. Generally, it is therefore advisable to operate the ion trap at rf amplitudes far below $100 \mathrm{~V}$ to significantly suppress the pockets. To overcome the pockets a significantly enhanced precision in the manufacturing and assembly of 22 pole traps is required. An interesting alternative for precisely controllable multipole ion traps are planar, chip-based traps 24].

We thank Ferdinand Schmidt-Kaler for helpful discussions. This work is supported by the Deutsche Forschungsgemeinschaft under contract No. WE 2592/2-1. P.H. acknowledges support by the Alexander von Humboldt foundation. K.S. acknowledges support by the European commission within EMALI (Contract No. MRTN-CT-2006-035369) and the Landesstiftung Baden-Württemberg in the framework atomics (Contract No. PN 63.14) and the Eliteprogramm Postdoktorandinnen und Postdoktoranden. 


\section{References}

[1] Gerlich D 1992 Adv. Chem. Phys. 821

[2] Gerlich D 1995 Phys. Scripta T59 256

[3] Schlemmer S, Kuhn T, Lescop E and Gerlich D 1999 Int. J. Mass Spectrom. 185-187 589

[4] Schlemmer S, Lescop E, von Richthofen J, Gerlich D and Smith M A 2002 J. Chem. Phys 117 2068

[5] Mikosch J, Kreckel H, Wester R, Plasil R, Glosik J, Gerlich D, Schwalm D and Wolf A $2004 J$. Chem. Phys. 12111030

[6] Asvany O, Kumar P, Redlich B, Hegemann I, Schlemmer S and Marx D 2005 Science 309 $1219-1222$

[7] Glosík J, Hlavenka P, Plašil R, Windisch F, Gerlich D, Wolf A and Kreckel H 2006 Phil. Trans. R. Soc. A 3642931

[8] Boyarkin O V, Mercier S R, Kamariotis A and Rizzo T R 2006 J. Am. Chem. Soc. 1282816

[9] Dzhonson A, Gerlich D, Bieske E J and Maier J P 2006 J. Mol. Struct. 795 93-97

[10] Kreckel H, Bing D, Reinhardt S, Petrignani A, Berg M and Wolf A 2008 J. Chem. Phys. 129 164312

[11] Asvany O, Savic I, Schlemmer S and Gerlich D 2004 Chem. Phys. 298 97-105

[12] Asvany O, Schlemmer S and Gerlich D 2004 Astroph. J. 617 685-692

[13] Otto R, Mikosch J, Trippel S, Weidemüller M and Wester R 2008 Phys. Rev. Lett. 101063201

[14] Wester R 2009 J. Phys. B submitted

[15] Dehmelt H G 1967 Adv. At. Mol. Phys. 353

[16] Schubert M, Siemers I and Blatt R 1989 J. Opt. Soc. Am. B 62159

[17] Walz J, Siemers I, Schubert M, Neuhauser W, Blatt R and Teloy E 1994 Phys. Rev. A 504122

[18] Mikosch J, Frühling U, Trippel S, Otto R, Hlavenka P, Schwalm D, Weidemüller M and Wester R 2008 Phys. Rev. A $\mathbf{7 8} 023402$

[19] Trippel S, Mikosch J, Berhane R, Otto R, Weidemüller M and Wester R 2006 Phys. Rev. Lett. 97193003

[20] Hlavenka P, Otto R, Trippel S, Mikosch J, Weidemüller M and Wester R 2009 J. Chem. Phys. 130061105

[21] Asvany O and Schlemmer S 2009 Int. J. Mass. Spectrom. 279147

[22] Greengard L 1988 The Rapid Evaluation of Potential Fields in Particle Systems (M.I.T. Press)

[23] Nabors K, Korsmeyer F T, Leighton F T and White J 1994 SIAM J. Sci. Stat. Comp. 15713

[24] Debatin M, Kröner M, Mikosch J, Trippel S, Morrison N, Reetz-Lamour M, Woias P, Wester R and Weidemüller M 2008 Phys. Rev. A 77033422 
a)

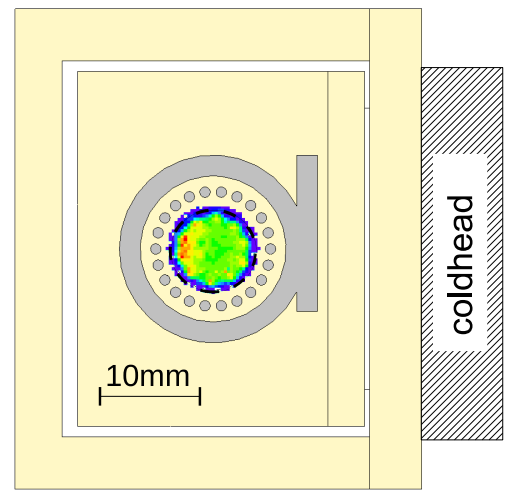

c)

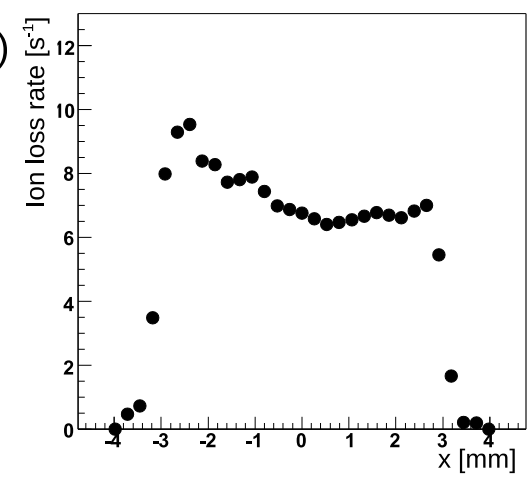

b)

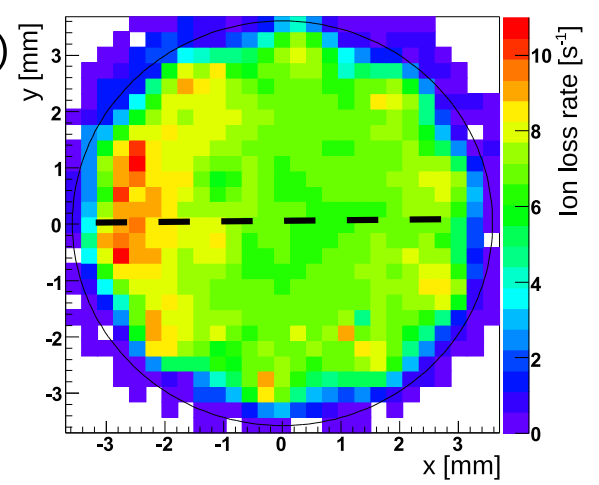

d)

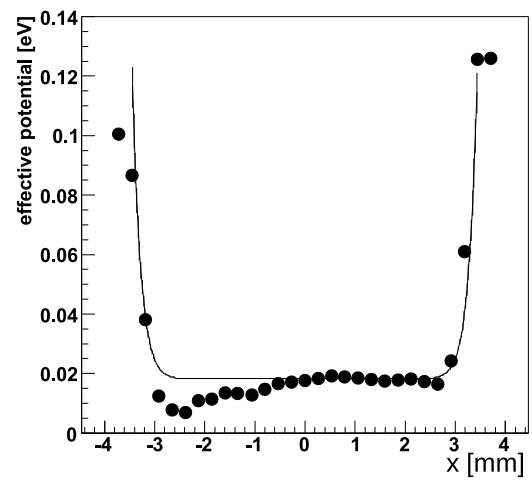

Figure 1. (Color online) a) Measured density distribution of trapped $\mathrm{OH}^{-}$ ions at $300 \mathrm{~K}$ buffer gas temperature with the rf amplitude set to $160 \mathrm{~V}$. The sketched geometry shows the layout of the ion trap, viewed along its symmetry axis. It includes the copper housing, the $22 \mathrm{rf}$ electrodes (end-on), a surrounding shaping electrode, and the end electrodes. b) Zoom into the measured ion density distribution, each pixel represents an individual decay rate measurement. c) One-dimensional cut through the density distribution along the horizontal axis. d) Effective potential derived from the density distribution by assuming a Boltzmanm distribution of the trapped ions at $300 \mathrm{~K}$. 

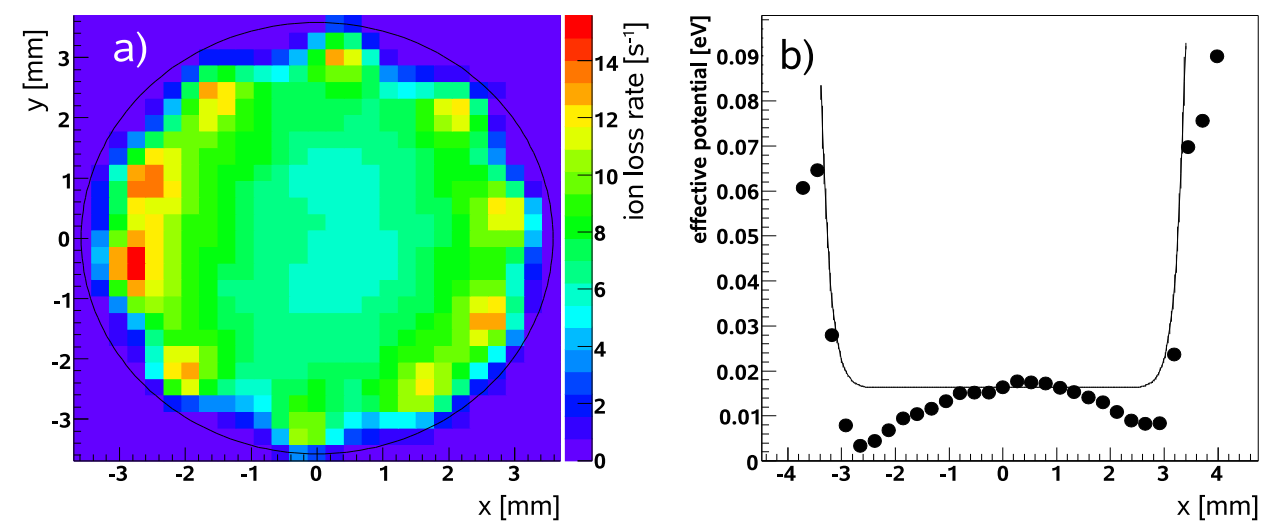

Figure 2. (Color online) a) Ion density distribution at $170 \mathrm{~K}$ with an $\mathrm{rf}$ amplitude of $160 \mathrm{~V}$. It shows a substructure of ten clearly distinct maxima. b) Cut through the effective potential, derived from the the density distribution. Overall, the potential is in accordance with the effective potential of an ideal 22-pole (solid line).
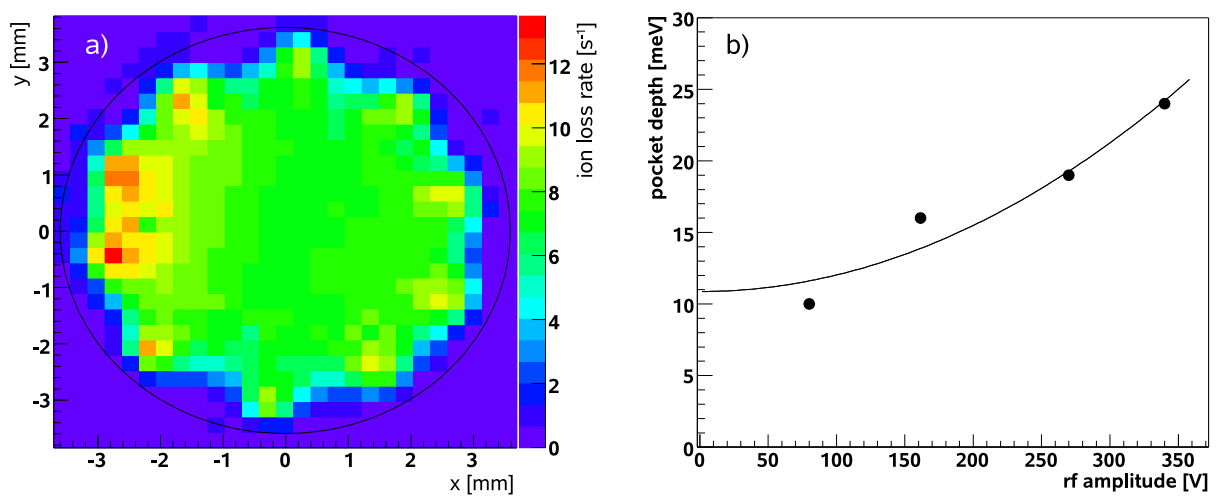

Figure 3. (Color online) a) Ion density distribution at $300 \mathrm{~K}$ with an rfamplitude of $270 \mathrm{~V}$. The histogram shows ten distinct maxima, which correspond to minima in the effective potential. b) The depth of the deepest minimum shows a strong increase as a function of the applied rf-amplitude. The solid line shows a polynomial fit with only a constant and a quadratic term. 

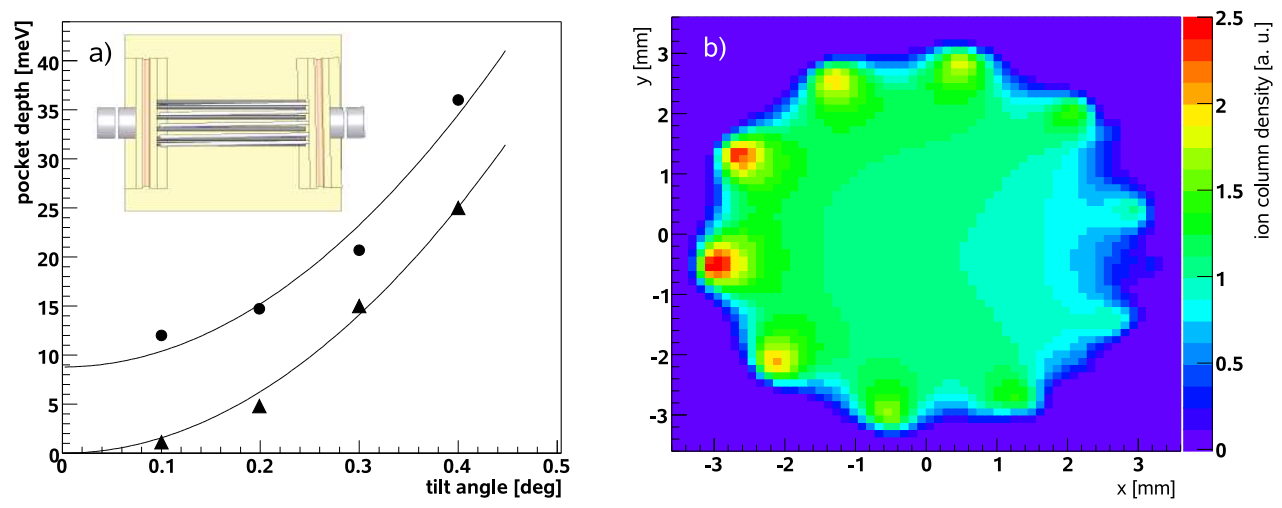

Figure 4. (Color online) a) Depth of the deepest potential minimum as a function of the tilt angle of the rf electrodes with a potential applied to the end electrodes (upper points) and without any potential on the end electrodes (lower points). The solid lines show quadratic fits without a linear term. The inset shows the geometry of the 22-pole ion trap, viewed in the direction onto the coldhead (see Fig. (17). The right wall of the trap together with the 11 implanted rf electrodes has been tilted by an angle of $1.0^{\circ}$. b) Calculated ion density distribution for $300 \mathrm{~K}$ in the effective potential of $270 \mathrm{~V} \mathrm{rf}$ amplitude and $-2 \mathrm{~V}$ static end electrode potential. A tilt angle of $0.15^{\circ}$ is chosen, which leads to a good agreement with the measured density distribution shown in Fig. 3 . 